\section{Consumo alimentar de macro e micronutrientes de crianças menores de cinco anos no Estado de Pernambuco, Brasil}

\section{Dietary intake of macro and micronutrients by children under five years of age in the State of Pernambuco, Brazil}

Cristianne Martins Ferreira Fidelis 1

Mônica Maria Osório2

1,2 Programa de Pós-Graduação em Nutrição. Centro de Ciências da Saúde. Universidade Federal de Pernambuco. Av Moraes Rego s.n. Cidade Universitária. Recife, PE, Brasil. CEP: 50.670-901. Email: mosorio@ufpe.br

\begin{abstract}
Objective: to analyze the food consumption of macro and micronutrients by children under fiver years old in the State of Pernambuco based on the Dietary Reference Intakes, in three geographic areas: Metropolitan Region of Recife, Urban Interior and Rural Interior.

Methods: the sample consisted of 948 children under five years old and was representative for the State of Pernambuco and its three geographic areas. The 24-hour recall method was used to register food consumption. The median and the prevalence of both macro and micronutrient inadequacy were analyzed according to the Dietary Reference Intakes.

Results: the deficit of energy and micronutrients prevailed in children of all age ranges and geographic areas; however, protein consumption exceeded the references levels. There was a high prevalence of micronutrient inadequacy, especially iron and zinc, in children above 12 months old. The results in the Rural Interior were the lowest, considering the nutrients analyzed.

Conclusions: food consumption by children in the State of Pernambuco is low in energy, macro and micronutrients. It is expected that these results may contribute to the implementation of health and nutrition policies at State level, aiming to prevent the main nutritional disturbances in children under five years old.
\end{abstract}

Key words Nutrients, Food consumption, Nutritional requirements, Child

\section{Resumo}

Objetivo: analisar o consumo alimentar de macro e micronutrientes, com base na Dietary Reference Intakes, das crianças menores de cinco anos do Estado de Pernambuco, considerando três áreas geográficas: Região Metropolitana do Recife, Interior Urbano e Interior Rural.

Métodos: a amostra constitui-se de 948 crianças menores de cinco anos de idade, com representatividade para o Estado de Pernambuco e suas três áreas geográficas. $O$ consumo alimentar foi registrado mediante o recordatório de 24 horas e foram analisadas as medianas e prevalências de inadequação dos macro e micronutrientes de acordo com as Dietary Reference Intakes.

Resultados: o déficit de energia e micronutrientes mostrou-se prevalente nas crianças, em todas as faixas etárias e áreas geográficas; entretanto, o consumo de proteínas apresentou-se acima dos valores de referência. A prevalência de inadequação dos micronutrientes foi elevada, principalmente de ferro e zinco, nas crianças acima de 12 meses de idade. Os resultados no Interior Rural foram os mais baixos para todos os nutrientes analisados.

Conclusões: o consumo alimentar das crianças do Estado de Pernambuco demonstrou déficit de energia, de macro e micronutrientes. Espera-se que estes resultados possam servir como subsídios para implementação de políticas de nutrição e alimentação no Estado, visando prevenir os principais distúrbios nutricionais em menores de cinco anos.

Palavras-chave Nutrientes, Consumo de alimentos, Necessidades nutricionais, Criança 


\section{Introdução}

Na criança, a nutrição adequada é fundamental para garantir crescimento e desenvolvimento normais e a manutenção de sua saúde, sendo este estágio de vida um dos biologicamente mais vulneráveis. ${ }^{1}$

Os nutrientes são constituintes dos alimentos necessários à manutenção das funções corporais normais. Esses compostos fornecem energia, macromoléculas (proteínas, lipídios e carboidratos), vitaminas, minerais, água, celulose e moléculas essenciais (aminoácidos e ácidos graxos essenciais). ${ }^{2}$

A inadequação de nutrientes interfere no processo de desenvolvimento e crescimento da criança e é fator determinante no aparecimento de carências nutricionais ou surgimento de várias manifestações patológicas que repercutirão na vida adulta, como doenças cardiovasculares, câncer, diabetes, etc. ${ }^{2}$ Quando o consumo de energia e de nutrientes está abaixo das necessidades, estabelecem-se as condições para o aparecimento das doenças carenciais que ainda acometem $40 \%$ da população humana. Ao contrário, se a oferta excede as exigências biológicas acima dos níveis toleráveis, a tendência é a instalação da chamada patologia dos excessos nutricionais. ${ }^{3}$

A ingestão de alimentos depende de fatores de ordem socioeconômica e cultural, podendo determinar situações cruciais na saúde do indivíduo. ${ }^{3} \mathrm{Em}$ países em desenvolvimento, como o Brasil, ainda existem prevalências marcantes de problemas nutricionais, principalmente em crianças pré-escolares, cujo determinante primário é o consumo alimentar. ${ }^{4}$ Essa população, muitas vezes, passa os primeiros anos de vida consumindo uma dieta monótona, a base de leite de vaca, farinha e açúcar, a qual contribui sobremaneira para o surgimento das carências nutricionais..$^{5-7}$ No Estado de Pernambuco, os dados das carências nutricionais ainda são elevados. A prevalência de desnutrição grave e moderada em crianças menores de 5 anos, segundo o índice peso/idade, é de $4,9 \%$, a anemia está presente em $40,9 \%$ das crianças e cerca de $19 \%$ apresentam hipovitaminose A.8,9

Dessa maneira, é de suma importância estabelecer, por meio dos estudos de consumo alimentar, as associações entre as condições de saúde e nutrição e a alimentação da população. Mesmo que sejam indicadores indiretos do estado nutricional, eles auxiliam no diagnóstico nutricional, tornando possível perceber um risco subclínico em potencial para o desencadeamento do problema, constituindose, assim, o primeiro indicador de risco nutricional. 10
Apesar da relevância do consumo alimentar, existem poucos estudos populacionais que identificam situações alimentares e nutricionais, especificamente no que se refere a vitaminas e minerais. $\mathrm{Na}$ tentativa de preencher essa lacuna, este estudo tem como objetivo analisar o consumo alimentar de macro e micronutrientes, com base na Ingestão Dietética Recomendada, das crianças menores de cinco anos do Estado de Pernambuco, considerando três áreas geográficas: Região Metropolitana do Recife, Interior Urbano e Interior Rural.

\section{Métodos}

Os dados analisados neste estudo foram coletados durante a II Pesquisa Estadual de Saúde e Nutrição (II PESN)8, em 1997, um estudo transversal de base domiciliar realizado no Estado de Pernambuco, Brasil, no período de fevereiro a maio de 1997, com amostras representativas de crianças menores de 5 anos e com o poder de desagregar por áreas geográficas, sendo elas: Região Metropolitana do Recife, Interior Urbano e Interior Rural.

Esta pesquisa foi desenvolvida pelo Departamento de Nutrição da Universidade Federal de Pernambuco (UFPE), Instituto Materno Infantil de Pernambuco (IMIP) e Secretaria Estadual de Saúde (SES) de Pernambuco e financiada pelo Instituto Nacional de Alimentação e Nutrição (extinto INAN) vinculado ao Ministério da Saúde. A II PESN/97 foi desenhada para atualizar e ampliar a Pesquisa Estadual de Saúde e Nutrição (PESN de 1991)11 - e teve como objetivo verificar a situação de saúde, alimentação e nutrição, e condição de vida da população materno-infantil. Na II PESN de 1997, a amostra foi dimensionada para garantir a representatividade de cada área geográfica, com estimativa da prevalência de $8,3 \%$ de desnutrição na Região Nordeste, tendo como base as tabulações iniciais da Pesquisa Nacional de Demografia e Saúde 12 e com um erro máximo de 2,2 pontos percentuais no valor previsto e nível de confiança de 95\%. Para isto, seriam necessárias no mínimo 600 crianças em cada uma das áreas, garantindo uma estimativa de prevalência esperada no intervalo de $6,1 \%$ a $10,5 \%$. O acréscimo de $10 \%$ nas amostras foi previsto para compensar as possíveis perdas ou problemas de não resposta. A amostra de fato pesquisada constou de valores próxi-mos aos da amostra proporcional necessária, tota-lizando 2081 crianças, sendo 751, 670 e 660, respectivamente na Região Metropolitana do Recife, Interior Urbano e Interior Rural. 


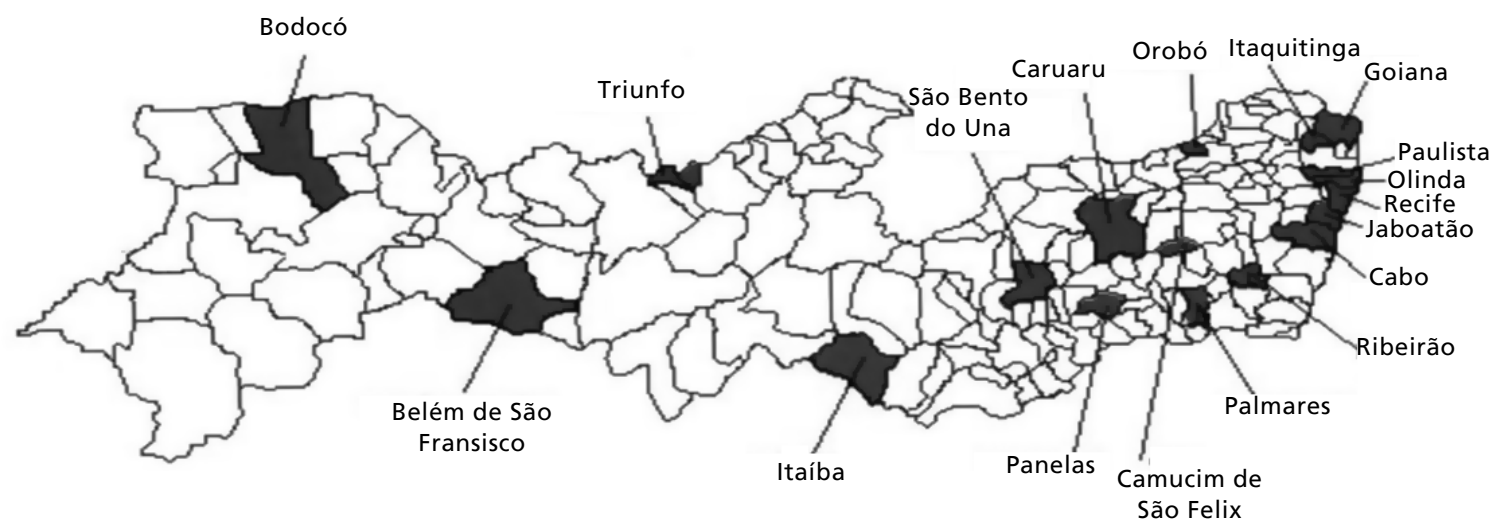

A amostra aleatória foi sorteada em três estágios: municípios, setores censitários e domicílios. Foram considerados na sua seleção os 18 municípios sorteados de forma aleatória simples na primeira pesquisa11 (Figura 1). Definiu-se, previamente, que seria investigado, em cada setor censitário, um total de 46 crianças, considerando-se a holística de campo em termos de número de entrevistadores e tempo disponível para a realização do trabalho. A partir dessa definição, foi feito um sorteio aleatório simples dos setores censitários, considerando a proporcionalidade da população de cada município, resultando 45 setores censitários. Por último, de posse do mapa do setor censitário, selecionou-se aleatoriamente uma quadra e em seguida uma esquina de cada setor, indicando a visita aos domicílios, um a um, em sentidohorário e identificando-se todas as crianças menores de cinco anos.

Para a realização do inquérito de consumo alimentar, um estudo inovador introduzido na II PESN, foi selecionado sistematicamente um terço das crianças menores de cinco anos, totalizando 992 crianças, assegurando representatividade para o Estado e suas três áreas geográficas. Os casos que apresentaram informações incompletas $(n=24)$ e as crianças menores de seis meses em aleitamento materno exclusivo $(\mathrm{n}=20)$ foram excluídos, resultando em 4,4\% de perdas. Após a limpeza dos dados, a amostra final constou de 948 crianças.

A coleta de campo foi realizada por uma equipe de entrevistadores composta por 14 profissionais de nível superior da área de saúde, subordinados a um supervisor de campo. Essa equipe recebeu previamente um treinamento de 40 horas. Um estudo piloto foi desenvolvido com aproximadamente 300 famílias, no município de Brejo da Madre Deus, localizado no Agreste Setentrional de Pernambuco, no qual, além de testar o instrumento de coleta, foi colocada em prática a logística do trabalho de campo, a fim de verificar a sua exequibilidade. Durante o trabalho de campo, o controle da qualidade das informações coletadas foi realizado pelo supervisor em $10 \%$ da amostra. As informações referentes ao consumo alimentar das crianças foram obtidas através do método recordatório de 24 horas, por meio de entrevista realizada com a mãe ou responsável pela criança. Utilizou-se um formulário próprio a fim de coletar todos os horários das refeições, alimentos e preparações, porções das preparações da família (quando a refeição da criança era a mesma da família), quantidade preparada, ofe recida e consumida pela criança. As mães ou responsáveis eram indagados sobre o tamanho das frutas e vegetais (pequeno, médio e grande); tamanho do prato e/ou da colher (pequeno, médio e grande); e quantidade de alimento (rasa, normal ou cheia) para alimentos preparados, leite, açúcar e massas; tamanho do copo ou xícara e a quantidade de líquidos contidos neles, para todos os líquidos. 8

Os alimentos, em medidas caseiras, foram pesados em laboratório, no total de cinco pesagens para cada porção ou medida caseira de cada alimento ou preparação. Posteriormente, foi obtida a média em gramas para cada medida caseira, a qual foi utilizada como padrão a ser adotado na digitação dos questionários. As dietas foram calculadas e analisadas utilizando o software "Virtual Nutri", desenvolvido por Philippi et al.,13 do Departamento de Nutrição da Faculdade de Saúde Pública da 
Tabela 1

\begin{tabular}{|c|c|c|c|c|c|}
\hline \multirow[t]{2}{*}{ Energia e Nutrientes } & \multirow[t]{2}{*}{ DRI's } & \multicolumn{4}{|c|}{ Estágio de vida } \\
\hline & & $0-6$ meses & 7-11 meses & $1-3$ anos & 4-8 anos \\
\hline Energia (kcal) & EER & 612 & 721 & 1051 & 1365 \\
\hline \multirow[t]{2}{*}{ Proteína (g) } & EAR & ** & 10 & 11 & 15 \\
\hline & $\mathrm{Al}$ & 9,1 & * & * & * \\
\hline \multirow[t]{2}{*}{ Carboidratos (g) } & EAR & ** & ** & 100 & ** \\
\hline & $\mathrm{Al}$ & 60 & 95 & * & * \\
\hline Gordura (g) & Al & 31 & 30 & * & * \\
\hline \multirow[t]{2}{*}{ Vitamina A $(\mu \mathrm{g})$} & EAR & ** & $* *$ & 210 & 275 \\
\hline & $\mathrm{Al}$ & 400 & 500 & * & * \\
\hline \multirow[t]{2}{*}{ Vitamina C (mg) } & EAR & ** & $* *$ & 13 & 22 \\
\hline & $\mathrm{Al}$ & 40 & 50 & * & * \\
\hline \multirow[t]{2}{*}{ Ferro (mg) } & EAR & ** & 6,9 & 3,0 & 4,1 \\
\hline & Al & 0,27 & * & * & * \\
\hline \multirow[t]{2}{*}{ Zinco (mg) } & EAR & ** & 2,5 & 2,5 & 4 \\
\hline & Al & 2 & * & * & * \\
\hline Cálcio (mg) & Al & 210 & 270 & 500 & 800 \\
\hline
\end{tabular}

Fonte: Institute of Medicine. National Research Council. Dietary Reference Intakes, 1999, 2002; *Valores de Al (dequate intake) não determinados; **Valores de EAR (recommended dietary allowances) não determinados

Universidade de São Paulo. Os alimentos que não constavam no programa foram inseridos a partir de tabelas de composição química de alimentos. $14,15 \mathrm{As}$ medianas, percentis e percentuais de risco de inadequação de energia e nutrientes foram obtidos através do programa Epi-Info, versão 6.04.

Para as análises das medianas e prevalências de inadequação dos macro e micronutrientes foram considerados os valores das Dietary Reference Intakes (DRI) (Tabela 1), propostos pelo Food and Nutrition Board (FND) ${ }^{16-19}$ sendo a amostra dividida em quatro faixas de idade (0-6 meses; 7-11 meses; 1-3 anos e de 4-5 anos).

As DRI baseiam-se no conceito de que a definição de necessidade nutricional representa o nível mínimo de ingestão de energia e nutrientes capaz de satisfazer as necessidades fisiológicas de grupos populacionais, acrescido de uma margem de segurança que excede a necessidade básica.
Abrangem quatro padrões de referência: Estimated Average Requeriment (EAR), Recommended Dietary Allowances (RDA), Adequate Intake (AI) e Tolerable Upper Intake Level (UL). Com a publicação das DRI, foi adquirido um novo conceito para o planejamento e a avaliação do consumo alimentar. ${ }^{20}$ A EAR é o valor da ingestão diária no qual se acredita suprir as necessidades de $50 \%$ dos indivíduos saudáveis de uma população e consiste na melhor forma de se estimar a prevalência do risco de inadequação de nutrientes. ${ }^{20} \mathrm{~A} \mathrm{AI}$ é a ingestão recomendada de nutrientes que se assume como adequada, sendo estabelecida a partir de estudos observacionais de indivíduos aparentemente sadios. Trata-se do nível de ingestão suficiente para alcançar ou exceder a quantidade necessária para a manutenção de um bom estado nutricional. É usada como meta de ingestão e está determinada para alguns nutrientes como o cálcio. 20 
As prevalências de inadequação de proteína, carboidrato, ferro, zinco e vitaminas A e C foram calculadas de acordo com a necessidade média estimada a EAR, considerando-se em risco a proporção de crianças do grupo que não alcançaram os valores de referência estabelecidos para o nutriente. 16-18 Nos nutrientes cuja EAR ainda não está estabelecida, como a gordura e o cálcio, não foi possível estimar a prevalência de risco de inadequação, sendo utilizada como referência a AI, verificando se a mediana de ingestão excedia ou não o valor de referência. $\mathrm{O}$ mesmo se aplica àqueles nutrientes cujo valor de EAR ainda não foi estabelecido para determinadas faixas etárias 16-19 (Tabela 1).

Para a avaliação da ingestão de energia foi utilizada a necessidade estimada de energia (Estimated Energy Requirement - EER), termo semelhante a EAR determinante do valor médio de ingestão de energia oriundo da dieta, necessário para manter o balanço energético para indivíduos saudáveis. Para calcular a EER, foram utilizadas as equações para predição de gasto energético total nos diferentes estágios de vida, considerando idade, sexo, peso, estatura e atividade física, adotando-se como coeficientes de atividade física os valores de 1,13 para o sexo masculino e de 1,16 para o sexo feminino, representando um nível de atividade física leve. 16

Considerando que o consumo de nutrientes difi- cilmente apresenta distribuição normal, todos os nutrientes foram calculados em valores medianos.

\section{Resultados}

A amostra deste estudo constou de 948 crianças, distribuídas entre as áreas geográficas, sendo 357 na Região Metropolitana do Recife, 297 no Interior Urbano e 294 no Interior Rural; e segundo a faixa etária, 145 entre 0-6 meses, 117 entre 7-11 meses, 570 entre 1-3 anos e 116 entre 4-5 anos, para o total do Estado. Com relação ao sexo, 50,2\% das crianças eram do sexo masculino e $49,8 \%$, do sexo feminino. A renda familiar mensal se situava em menos de 2 salários mínimos para $46,1 \%$ das suas famílias.

A Tabela 2 apresenta as medianas, percentis (25 e 75) e os percentuais de risco de inadequação do consumo alimentar das crianças menores de cinco anos do Estado de Pernambuco. Observam-se acentuadas prevalências de inadequação no consumo de energia, principalmente nas faixas etárias de 0-6 meses $(49 \%)$ e de $4-5$ anos $(55,2 \%)$, embora as medianas de consumo estivessem acima das necessidades energéticas estimadas (EER), exceto para as crianças maiores de quatro anos, onde a mediana não alcançou a EER. O consumo mediano de proteína e carboidrato manteve-se acima das necessidades médias estimadas (EAR). Nas faixas etárias menores

Tabela 2

Consumo mediano, percentis e prevalência de inadequação de energia e nutrientes em crianças menores de cinco anos. Pernambuco, 1997.

\begin{tabular}{|c|c|c|c|c|c|c|c|c|c|c|c|c|c|c|c|c|}
\hline \multirow[t]{3}{*}{ Nutrientes } & \multicolumn{5}{|c|}{$0-6$ meses (145) } & \multicolumn{3}{|c|}{ 7-11 meses (117) } & \multicolumn{5}{|c|}{$1-3$ anos $(570)$} & \multicolumn{3}{|c|}{ 4-5 anos (116) } \\
\hline & Md & $\mathrm{Pe}$ & $\mathrm{Pe}$ & $\%$ & $\mathrm{Md}$ & $\mathrm{Pe}$ & $\mathrm{Pe}$ & $\%$ & $\mathrm{Md}$ & $\mathrm{Pe}$ & $\mathrm{Pe}$ & $\%$ & Md & $\mathrm{Pe}$ & $\mathrm{Pe}$ & $\%$ \\
\hline & & 25 & 75 & inad. & & 25 & 75 & inad. & & 25 & 75 & inad. & & 25 & 75 & inad. \\
\hline Energia (kcal) & 626,2 & 483,4 & 910,9 & $49,0^{a}$ & 969,2 & 961,1 & 1329,2 & $26,5^{a}$ & 1156,8 & 859,2 & 1486,3 & $41,4^{a}$ & 1274,8 & 931,4 & 1746,8 & $55,2^{a}$ \\
\hline Proteína (g) & 16,2 & 8,0 & 29,1 & * & 30,2 & 19,6 & 41,5 & 6,0 & 37,2 & 26,0 & 52,9 & 4,4 & 43,3 & 30,8 & 57,9 & 6,9 \\
\hline Carboidrato (g) & 78,8 & 51,5 & 132,7 & * & 137,8 & 94,5 & 198,2 & * & 156,4 & 117,4 & 212,6 & 16,8 & 186,6 & 126,0 & 252,0 & $* *$ \\
\hline Gordura (g) & 27,8 & 19,6 & 34,7 & * & 29,7 & 21,5 & 40,4 & * & 35,4 & 23,9 & 49,0 & $* *$ & 36,9 & 25,7 & 57,4 & $* *$ \\
\hline Vitamina A $(\mu \mathrm{g})$ & 414,2 & 139,4 & 557,3 & * & 314,8 & 98,5 & 739,9 & * & 249,0 & 91,5 & 492,3 & 44,0 & 207,1 & 100,4 & 428,7 & 59,5 \\
\hline Vitamina C (mg) & 33,8 & 16,6 & 51,3 & * & 31,3 & 13,1 & 97,6 & * & 36,6 & 13,7 & 97,8 & 23,2 & 41,7 & 14,4 & 89,2 & 36,2 \\
\hline Ferro $(\mathrm{mg})$ & 2,3 & 0,6 & 5,3 & * & 4,6 & 2,0 & 8,8 & 65,0 & 5,9 & 3,3 & 9,4 & 23,7 & 7,0 & 4,5 & 10,0 & 22,4 \\
\hline Zinco (mg) & 1,3 & 0,3 & 2,8 & * & 1,8 & 0,4 & 4,4 & 57,3 & 3,1 & 1,3 & 5,4 & 43,7 & 3,6 & 1,9 & 6,3 & 52,6 \\
\hline Cálcio (mg) & 393,5 & 216,7 & 801,9 & * & 739,6 & 302,0 & 1171,3 & $*$ & 522,1 & 183,7 & 916,2 & * & 282,2 & 125,1 & 644,7 & * \\
\hline
\end{tabular}

a=valores de EER (Estimated Energy Requirement) calculados; *valores de DRI (Dietary reference intake) determinado por AI (Adequate Intake); **valores de DRI não determinados; Demais nutrientes = EAR (Recommended Dietary Allowances); Md = mediana; Pe = percentil; Inad. = Inadequado. 
Tabela 3

Consumo mediano, percentis e prevalência de inadequação de energia e nutrientes em crianças menores de cinco anos. Região Metropolitana do Recife, Pernambuco, 1997.

\begin{tabular}{|c|c|c|c|c|c|c|c|c|c|c|c|c|c|c|c|c|}
\hline \multirow[t]{3}{*}{ Nutrientes } & \multicolumn{5}{|c|}{ 0-6 meses (52) } & \multicolumn{3}{|c|}{ 7-11 meses (42) } & \multicolumn{5}{|c|}{$1-3$ anos (219) } & \multicolumn{3}{|c|}{ 4-5 anos (44) } \\
\hline & $\mathrm{Md}$ & $\mathrm{Pe}$ & $\mathrm{Pe}$ & $\%$ & Md & $\mathrm{Pe}$ & $\mathrm{Pe}$ & $\%$ & $\mathrm{Md}$ & $\mathrm{Pe}$ & $\mathrm{Pe}$ & $\%$ & Md & $\mathrm{Pe}$ & $\mathrm{Pe}$ & $\%$ \\
\hline & & 25 & 75 & inad. & & 25 & 75 & inad. & & 25 & 75 & inad. & & 25 & 75 & inad. \\
\hline Energia (kcal) & 586,0 & 452,7 & 799,9 & $55,8^{a}$ & 1123,0 & 745,6 & 1612,6 & $21,4^{a}$ & 1224,0 & 878,4 & 1527,6 & $39,3^{a}$ & 1314,4 & 1025,0 & 1822,9 & $52,3^{a}$ \\
\hline Proteína (g) & 15,0 & 8,3 & 30,2 & * & 34,1 & 20,6 & 47,0 & 0,0 & 39,6 & 27,4 & 55,4 & 4,1 & 49,0 & 34,2 & 61,2 & 2,3 \\
\hline Carboidratos (g) & 78,7 & 54,2 & 118,8 & * & 169,4 & 95,5 & 217,9 & * & 156,4 & 122,8 & 212,6 & 13,2 & 182,7 & 136,9 & 248,7 & $* *$ \\
\hline Gordura (g) & 26,7 & 17,4 & 32,9 & * & 32,7 & 22,0 & 43,8 & * & 37,5 & 24,7 & 53,1 & $* *$ & 38,8 & 32,7 & 60,2 & $* *$ \\
\hline Vitamina $A(\mu \mathrm{g})$ & 469,0 & 305,1 & 924,3 & * & 643,3 & 355,6 & 1806,7 & * & 370,8 & 194,5 & 710,4 & 27,9 & 350,1 & 212,3 & 680,3 & 43,2 \\
\hline Vitamina C (mg) & 50,0 & 28,9 & 98,5 & * & 95,5 & 41,9 & 199,1 & * & 59,4 & 25,1 & 142,2 & 12,3 & 62,1 & 14,8 & 100,4 & 36,2 \\
\hline Ferro (mg) & 2,4 & 0,5 & 6,0 & * & 8,8 & 4,9 & 11,9 & 38,1 & 7,0 & 4,5 & 11,1 & 15,5 & 7,9 & 5,2 & 12,0 & 22,4 \\
\hline Zinco (mg) & 1,2 & 0,6 & 2,8 & * & 3,4 & 0,8 & 5,2 & 33,3 & 3,6 & 1,8 & 6,2 & 34,2 & 4,4 & 2,4 & 6,3 & 52,6 \\
\hline Cálcio (mg) & 506,4 & 240,7 & 777,4 & * & 807,6 & 474,8 & 1259,8 & * & 607,7 & 335,7 & 986,0 & * & 437,7 & 167,2 & 733,2 & * \\
\hline
\end{tabular}

$a=$ valores de EER (Estimated Energy Requirement) calculados; *valores de DRI (Dietary reference intake) determinado por AI (Adequate Intake); **valores de DRI não determinados; Demais nutrientes = EAR (Recommended Dietary Allowances); Md = mediana; Pe = percentil; Inad.=Inadequado.

de 12 meses, as suas medianas de consumo também se encontravam acima da ingestão adequada (AI). O consumo mediano de gordura não alcançou o valor da AI nas faixas etárias de menores de um ano.

Com relação aos micronutrientes, a vitamina A apresentou elevados percentuais de inadequação, $44 \%$ e $59,5 \%$ nas faixas etárias acima de 11 meses, embora nas crianças de um a três anos a mediana tenha sido acima da EAR. Na faixa etária de 7-11 meses, a mediana de consumo de vitamina A se manteve abaixo da AI. O consumo de vitamina $\mathrm{C}$ foi bastante superior a EAR nas faixas etárias a partir de um ano e inferior a AI nas idades menores de um ano. Chamam a atenção os valores de inadequação do ferro, principalmente na faixa etária de 7-11 meses $(65 \%)$, em que a mediana de consumo foi menor do que a EAR. Para os menores de seis meses, a mediana do consumo de ferro esteve acima da AI. $\mathrm{O}$ zinco mostrou percentuais elevados de inadequação nas faixas etárias de 7-11 meses, um a três anos e de um a quatro anos, correspondendo a $57,3 \%, 43,7 \%$ e $52,6 \%$, respectivamente, apesar de a faixa etária de um a três anos ter apresentado a mediana acima da EAR. O consumo mediano de zinco nos menores de seis meses encontrava-se abaixo da AI. O cálcio apresentou a mediana de consumo abaixo da AI apenas na faixa etária de quatro a cinco anos.
Na Região Metropolitana do Recife, foram observados elevados percentuais de inadequação de energia, principalmente nas faixas etárias de zero a seis meses $(55,8 \%)$ e de quatro a cinco anos $(52,3 \%)$, em que as medianas não alcançaram a EER, (Tabela $3)$. Os valores medianos de proteína e carboidrato superaram a EAR, revelando baixos percentuais de inadequação desses nutrientes. As medianas de proteína e de carboidrato também foram superiores a AI nas crianças de zero a seis meses. $\mathrm{O}$ consumo mediano de gordura estava abaixo da AI apenas nas crianças menores de seis meses. As medianas do consumo de vitamina $\mathrm{A}$ e de vitamina $\mathrm{C}$ superaram a EAR e a AI para as faixas de idade correspondentes, embora, nas faixas etárias a partir de um ano tenham sido encontrados elevados percentuais de inadequação, com exceção da mediana de vitamina $C$ na faixa etária de um a três anos. $\mathrm{O}$ ferro e o zinco apresentaram elevadas prevalências de inadequação, principalmente o zinco, nas crianças de quatro anos e mais de idade $(52,6 \%)$ e o ferro nas crianças de 7 11 meses $(38,1 \%)$. Nas crianças menores de seis meses, a mediana do consumo de ferro foi superior a $\mathrm{AI}$, e a de zinco, inferior. O cálcio apresentou a mediana de consumo abaixo da AI apenas na faixa etária acima de quatro a cinco anos.

No Interior Urbano, a inadequação de energia variou de $28,6 \%$ (7-11meses) a 42,9\% (4-5 anos), 
Consumo mediano, percentis e prevalência de inadequação de energia e nutrientes em crianças menores de cinco anos. Interior Urbano, Pernambuco, 1997.

\begin{tabular}{|c|c|c|c|c|c|c|c|c|c|c|c|c|c|c|c|c|}
\hline \multirow[t]{3}{*}{ Nutrientes } & \multicolumn{5}{|c|}{$0-6$ meses $(53)$} & \multicolumn{3}{|c|}{ 7-11 meses (35) } & \multicolumn{5}{|c|}{ 1-3 anos (174) } & \multicolumn{3}{|c|}{ 4-5 anos (35) } \\
\hline & Md & $\mathrm{Pe}$ & $\mathrm{Pe}$ & $\%$ & Md & $\mathrm{Pe}$ & $\mathrm{Pe}$ & $\%$ & Md & $\mathrm{Pe}$ & $\mathrm{Pe}$ & $\%$ & Md & $\mathrm{Pe}$ & $\mathrm{Pe}$ & $\%$ \\
\hline & & 25 & 75 & inad. & & 25 & 75 & inad. & & 25 & inad. & & & 75 & inad. & \\
\hline Energia (kcal) & 694,8 & 515,5 & 942,4 & $39,6^{a}$ & 886,0 & 638,4 & 1162,2 & $28,6^{a}$ & 1226,3 & 964,9 & 1633,0 & $29,9 a$ & 1501,4 & 947,6 & 1941,4 & $42,9^{a}$ \\
\hline Proteína (g) & 20,5 & 10,8 & 29,2 & * & 29,9 & 19,0 & 41,2 & 11,4 & 40,5 & 29,4 & 54,2 & 2,9 & 49,0 & 32,3 & 58,4 & 2,9 \\
\hline Carboidratos (g) & 82,8 & 49,3 & 130,9 & * & 121,9 & 101,6 & 180,1 & * & 169,8 & 125,0 & 239,6 & 11,5 & 215,8 & 114,8 & 274,3 & $* *$ \\
\hline Gordura (g) & 27,1 & 117,4 & 35,6 & * & 27,9 & 22,6 & 37,1 & * & 39,5 & 25,6 & 51,0 & $* *$ & 43,4 & 28,9 & 64,8 & $* *$ \\
\hline Vitamina A $(\mu \mathrm{g})$ & 224 & 44,5 & 557,3 & * & 232,7 & 88,4 & 615,6 & * & 220,6 & 69,6 & 484,0 & 48,9 & 208,1 & 100,6 & 429,5 & 54,3 \\
\hline Vitamina C (mg) & 23,1 & 6,5 & 44,5 & * & 27,3 & 14,2 & 90,5 & * & 46,9 & 21,3 & 124,5 & 17,8 & 45,2 & 15,9 & 93,2 & 31,4 \\
\hline Ferro (mg) & 2,8 & 1,4 & 6,2 & * & 4,2 & 2,2 & 5,5 & 77,1 & 7,1 & 4,4 & 10,9 & 14,4 & 8,3 & 4,8 & 10,4 & 14,3 \\
\hline Zinco (mg) & 1,3 & 0,0 & 3,2 & * & 1,2 & 0,4 & 3,9 & 71,4 & 3,4 & 1,4 & 5,8 & 41,4 & 4,5 & 2,3 & 8,1 & 40,0 \\
\hline Cálcio (mg) & 443,9 & 220,6 & 955,8 & * & 736,3 & 195,4 & 1049,4 & * & 626,2 & 268,5 & 971,8 & * & 272,2 & 138,5 & 681,8 & * \\
\hline
\end{tabular}

$\mathrm{a}=$ valores de EER (Estimated Energy Requirement) calculados; *valores de DRI (Dietary reference intake) determinado por Al (Adequate Intake); **valores de DRI não determinados; Demais nutrientes = EAR (Recommended Dietary Allowances); $M d=$ mediana; $P e=$ percentil; Inad.=Inadequado.

embora as medianas de consumo tenham se estabelecido acima da EER (Tabela 4). As medianas de consumo de proteína se mantiveram acima da EAR e AI, com baixos percentuais de inadequação, cujo valor máximo foi de $11,4 \%$ na faixa etária de 7-11 meses. O consumo mediano de carboidrato se manteve acima das AI e EAR, com baixa prevalência de inadequação nas crianças de um a três anos $(11,5 \%)$. O consumo de gordura nas crianças menores de um ano não alcançou a AI. O consumo de vitamina A foi superior a EAR na faixa etária de um a três anos e inferior na de quatro a cinco anos, com percentuais de inadequação em torno de $50 \%$. As suas medianas não alcançaram a AI nas crianças menores de 12 meses. O consumo de vitamina C superou bastante a EAR nas faixas etárias a partir de um ano, entretanto os percentuais de inadequação foram de $17,8 \%$ e $31,4 \%$, respectivamente, para as crianças de um a três anos e quatro a cinco anos. As medianas de consumo de vitamina $\mathrm{C}$, quando comparadas com a AI, apresentaram valores inferiores a esta. Os resultados para os minerais apontam valores muitos elevados de inadequação de ferro e zinco, alcançando $77,1 \%$ e $71,4 \%$, respectivamente, nas crianças de 7-11 meses, sendo essa a única faixa etária que apresentou consumo mediano abaixo da EAR. De acordo com a AI, o consumo de ferro e de zinco estabeleceu-se acima e abaixo da recomen- dação, respectivamente. O consumo de cálcio não atingiu a $\mathrm{AI}$ apenas nas crianças maiores de quatro anos.

O Interior Rural, quando comparado às demais áreas geográficas, apresentou, de maneira geral, os maiores percentuais de inadequação de consumo em todos os nutrientes e faixas etárias (Tabela 5). As medianas do consumo de energia, ao contrário das outras áreas geográficas, mantiveram-se abaixo da EER, com exceção da faixa etária de 7-11 meses. As prevalências de inadequação de energia foram, em geral, elevadas, superando $70 \%$ nas crianças de quatro a cinco anos. As medianas de proteína estavam acima dos valores da EAR e AI. O consumo mediano de carboidrato se manteve acima da EAR e AI, com a prevalência de inadequação de $26,6 \%$ nas crianças de um a três anos. O consumo de gordura não alcançou a AI para os menores de um ano. A vitamina A apresentou medianas de consumo abaixo da EAR e AI, exceto nas crianças menores de seis meses, e elevadas prevalências de inadequação, principalmente nas crianças de $4-5$ anos $(83,8 \%)$. A vitamina $\mathrm{C}$ apresentou consumo mediano acima da EAR, porém com prevalências de inadequação acima de $40 \%$, e abaixo da AI, para as faixas etárias menores de um ano. $\mathrm{O}$ ferro e o zinco também apresentaram altas prevalências de inadequação, principalmente na faixa etária de 7-12 meses, com 82,5\% 
Tabela 5

Consumo mediano, percentis e prevalência de inadequação de energia e nutrientes em crianças menores de cinco anos no Interior Rural, Pernambuco, 1997.

\begin{tabular}{|c|c|c|c|c|c|c|c|c|c|c|c|c|c|c|c|c|}
\hline \multirow[t]{3}{*}{ Nutrientes } & \multicolumn{5}{|c|}{$0-6$ meses $(40)$} & \multicolumn{3}{|c|}{$7-11$ meses $(40)$} & \multicolumn{5}{|c|}{$1-3$ anos (177) } & \multicolumn{3}{|c|}{ 4-5 anos (37) } \\
\hline & Md & $\mathrm{Pe}$ & $\mathrm{Pe}$ & $\%$ & Md & $\mathrm{Pe}$ & $\mathrm{Pe}$ & $\%$ & Md & $\mathrm{Pe}$ & $\mathrm{Pe}$ & $\%$ & Md & $\mathrm{Pe}$ & $\mathrm{Pe}$ & $\%$ \\
\hline & & 25 & 75 & inad. & & 25 & 75 & inad. & & 25 & 75 & inad. & & 25 & 75 & inad. \\
\hline Energia (kcal) & 606,4 & 484,6 & 964,5 & $52,5^{a}$ & 964,1 & 627,4 & 1172,2 & $26,5^{a}$ & 982,4 & 711,9 & 1302,4 & $55,4^{a}$ & 1045,2 & 867,3 & 1462,1 & $70,3^{a}$ \\
\hline Proteína (g) & 11,5 & 7,2 & 25,1 & * & 29,2 & 18,7 & 39,9 & 7,5 & 30,8 & 21,6 & 46,3 & 6,2 & 33,9 & 22,3 & 57,8 & 16,2 \\
\hline Carboidratos (g) & 76,0 & 48,7 & 163,6 & * & 117,0 & 92,1 & 159,6 & * & 139,8 & 97,4 & 185,2 & 26,6 & 164,4 & 117,2 & 206,3 & $* *$ \\
\hline Gordura (g) & 29,1 & 23,3 & 34,4 & * & 27,9 & 20,8 & 38,6 & * & 28,9 & 22,6 & 40,2 & $* *$ & 25,5 & 20,7 & 45,4 & $* *$ \\
\hline Vitamina A $(\mu \mathrm{g})$ & 414,2 & 192,5 & 498,5 & * & 153,3 & 40,4 & 323,7 & * & 155,8 & 52,7 & 310,5 & 59,3 & 121,5 & 63,4 & 176,2 & 83,8 \\
\hline Vitamina C (mg) & 32,7 & 20,4 & 39,0 & * & 13,7 & 8,0 & 28,3 & * & 16,0 & 6,5 & 33,9 & 41,8 & 23,6 & 10,7 & 51,2 & 45,9 \\
\hline Ferro (mg) & 1,0 & 0,2 & 3,7 & * & 2,3 & 1,1 & 5,5 & 82,5 & 3,7 & 1,9 & 6,5 & 42,9 & 5,4 & 3,4 & 9,1 & 35,1 \\
\hline Zinco (mg) & 1,2 & 0,7 & 1,8 & * & 1,2 & 0,2 & 3,2 & 70,0 & 2,1 & 0,8 & 4,0 & 57,6 & 2,2 & 1,3 & 5,0 & 70,3 \\
\hline Cálcio (mg) & 259,1 & 206,1 & 573,0 & * & 574,7 & 219,3 & 1252,1 & * & 246,8 & 69,9 & 710,6 & * & 166,9 & 86,8 & 502,3 & * \\
\hline
\end{tabular}

$a=$ valores de EER (Estimated Energy Requirement) calculados; *valores de DRI (Dietary reference intake) determinado por AI (Adequate Intake); **valores de DRI não determinados; Demais nutrientes = EAR (Recommended Dietary Allowances); Md = mediana; Pe = percentil; Inad. = Inadequado.

de inadequação para o ferro. Entretanto, a mediana de consumo de ferro manteve-se acima da EAR, exceto para as crianças de 7-11 meses, e acima da AI. Para o zinco, todas as medianas estiveram abaixo da EAR e AI, nas crianças de 7-11 meses e de 4-5 anos a inadequação se estabeleceu em torno de $70 \%$. A mediana de cálcio não atingiu a AI nas faixas etárias de maiores de um ano.

\section{Discussão}

Esta pesquisa representa o primeiro estudo de base populacional realizado no Estado de Pernambuco de avaliação do consumo alimentar de macro e micronutrientes das crianças menores de cinco anos. A I Pesquisa Estadual de Saúde e Nutrição (PESN) em 1991, foi pioneira na realização de um estudo abrangente da situação de saúde e nutrição das crianças do Estado, entretanto, não avaliou o consumo alimentar dessa população. A II Pesquisa Estadual de Saúde e Nutrição, (II PESN de 1997) 8 ampliou o estudo no sentido de conhecer o perfil alimentar das crianças, realizando o inquérito dietético, por meio do método recordatório de 24 horas, em amostras representativas para cada área geográfica (Região Metropolitana do Recife, Interior Urbano e Interior Rural).
Conhecer exatamente a ingestão alimentar de indivíduos ou de grupos é sempre uma tarefa complexa. Os aspectos culturais e até as experiências pessoais, nos métodos de investigação sobre consumo alimentar, conferem menos objetividade nos resultados do que se espera. Cada método empregado para avaliar o consumo alimentar apresenta vantagens e limitações e os estudos requerem uma equipe qualificada e capacitada para a coleta dos dados. Como estimativa da realidade do consumo alimentar, o recordatório de 24 horas ainda se constitui o método mais apropriado para avaliar a ingestão de alimentos de uma população. Entretanto, cabe salientar que esse método, ao ser utilizado para verificar o consumo alimentar atual de crianças, por problemas de sub-registro na quantificação das informações, pode subestimar a ingestão energética em torno de $30 \%$ e, por conseguinte, superestimar os percentuais de prevalência de inadequação de nutrientes. 10,21 Além disso, o recordatório de 24 horas, sendo um método retrospectivo para a avaliação da dieta, pode ocasionar vieses de memória, uma vez que o entrevistado tem que recordar a sua ingestão alimentar do dia anterior à entrevista. 10

De um modo geral, a mediana do consumo de energia das crianças no Estado de Pernambuco manteve-se acima da EER para todas as faixas etárias. Entretanto, quando se avalia a prevalência de 
inadequação, observa-se que os percentuais são acentuados, sendo as crianças menores de seis meses e as maiores de quatro anos as mais prejudicadas. $\mathrm{O}$ primeiro caso poderia ser explicado pela introdução precoce e inadequada da alimentação complementar, com baixa ingestão de alimentos fontes de energia, ricos em carboidratos e gordura, embora um estudo anterior na mesma população tenha demonstrado que nas crianças menores de seis meses, os alimentos mais freqüentemente consumidos eram leite, leite materno, açúcar, amido de milho e alimentos a base de cereal. ${ }^{5}$ Isso, no entanto, não significa que os mesmos eram consumidos em quantidades adequadas, sugerindo assim, uma explicação para as elevadas prevalências de inadequação. No segundo caso, o estudo anterior demonstra que o aumento da idade da criança faz com que diminua o consumo de alimentos a base de leite, amido de milho e de cereais, ainda que tenha existido um aumento no consumo de gordura, biscoito, pão, batata e milho. Mas, da mesma maneira, não significa que o consumo tenha sido suficiente para atender as necessidades das crianças. Assis e Barreto6 encontraram resultados semelhantes em Salvador, nas crianças da mesma faixa etária (48-60 meses) e sugeriram que o motivo para a inadequação nessa idade se devia ao fato de que as crianças deixavam de realizar algumas das refeições.

Analisando comparativamente cada área geográfica, o Interior Urbano apresentou as medianas de consumo de energia acima das EER para todas as faixas etárias e as prevalências de inadequação foram as menores, embora os alimentos mais freqüentemente consumidos tenham sido semelhantes aos das outras áreas. 5 No Interior Rural, as medianas do consumo de energia não alcançaram as EER, com exceção da faixa de 7-11 meses de idade. Essa foi a área de maior prevalência de inadequação energética, principalmente nas crianças maiores de 4 anos $(70,3 \%)$. Esses resultados podem se refletir no estado nutricional das crianças, uma vez que Pernambuco apresenta uma desnutrição moderada/grave de $4,9 \%$ e $12,1 \%$, respectivamente pelos índices peso/idade e altura/idade, sendo o Interior Rural a área de maior prevalência. ${ }^{8}$

O consumo mediano de proteína no Estado manteve-se sempre acima do recomendado, chegando mesmo a triplicar em algumas faixas de idade, com comportamento semelhante em todas as áreas geográficas, inclusive no Interior Rural. Ainda assim, as prevalências de inadequação foram mais expressivas nessa área geográfica, principalmente nas crianças maiores de quatro anos.

De uma forma geral, a proteína alcançou media- nas de consumo bem mais elevadas do que a de energia, corroborando os dados do Brasil, e particularmente do Nordeste, que sugerem o déficit de energia mais freqüente do que o déficit protéico. ${ }^{22} \mathrm{~A}$ proteína é uma macromolécula por função plástica e constitui mais da metade do peso seco de vários órgãos, participando de processos orgânicos vitais e mantendo o balanço nitrogenado positivo. $\mathrm{O}$ desvio de sua função principal pode acarretar déficit de crescimento, tornando-se preocupante a possibilidade do componente mais caro da dieta, na insuficiência de outras fontes, ser utilizado pelo organismo como fonte primária de energia. ${ }^{2}$ Essa possibilidade é factível tendo em vista as significativas prevalências de inadequação de carboidrato e de crianças que não alcançaram a recomendação de gordura.

As vitaminas e os minerais são nutrientes não energéticos que não podem ser sintetizados no corpo humano, devendo assim, serem supridos pela alimentação. Os minerais desempenham uma variedade expressiva de funções metabólicas que incluem ativação, regulação, transmissão e controle; e as vitaminas possuem funções relacionadas com as atividades estruturais. Portanto, esses micronutrientes são importantes para o crescimento e desenvolvimento saudável da criança e suas deficiências podem causar seqüelas irreparáveis. As carências de minerais e vitaminas aparecem em estágio subclínico, sem que haja comprometimento do estado nutricional, detectado pela avaliação antropométrica, podendo se agravar com o surgimento de diversos sinais e sintomas. 23

A Vitamina A é essencial à manutenção das funções fisiológicas do organismo, participa do ciclo visual, da integridade das membranas biológicas, da manutenção e diferenciação epitelial, atua na formação de glicoproteínas e na resposta imune. O papel da vitamina A no sistema imunológico aumentar a imunidade humoral, a concentração de anticorpos ativos, o número de células epiteliais formadoras de anticorpos e a imunidade local, o que pode repercutir na diminuição da morbidade por diarréia e infecção respiratória aguda. ${ }^{24}$ Uma deficiência no consumo de alimentos fontes de vitamina A e seus precursores, os carotenóides, explicaria a elevada prevalência de sua inadequação encontrada em todas as áreas geográficas e em todas as faixas etárias, principalmente nas crianças maiores de 4 anos. Embora a vitamina A disponível na dieta também seja fornecida pelo leite, parece mais uma vez que a quantidade não tem sido suficiente para suprir as necessidades diárias. Essas crianças também apresentavam um consumo bastante reduzido de vísceras e vegetais ricos em 
carotenóides. ${ }^{5}$ Salienta-se ainda o fato de que a conversão de carotenóides em vitamina A depende da presença de outros componentes da dieta, como a gordura e a vitamina $\mathrm{E}$, e da interação com outros nutrientes. ${ }^{24}$ É reconhecido que a vitamina A atua no mecanismo da absorção do ferro e, conseqüentemente, sua deficiência pode determinar o aparecimento da anemia. 25

Os resultados de inadequação de vitamina $\mathrm{A}$ parecem repercutir nos níveis de retinol sérico nesta mesma população, em que $19,3 \%$ das crianças apresentavam esses níveis abaixo de $20 \mathrm{mcg} / \mathrm{dl}$ e $3 \%$ abaixo de $10 \mathrm{mcg} / \mathrm{dl}$, indicando hipovitaminose $\mathrm{A}$, caracterizando um problema de saúde pública de leve a moderado. ${ }^{8}$

O consumo mediano de vitamina $\mathrm{C}$ nas crianças menores de um ano de idade estabeleceu-se abaixo das recomendações no Interior Rural. Mesmo o consumo de vitamina $\mathrm{C}$ excedendo as EAR nas demais faixas etárias, não se pode garantir seu bom aproveitamento pelo organismo, pois, geralmente os alimentos ricos nesse nutriente, em geral as frutas e hortaliças, são oferecidos em pequena quantidade e/ou cozidas na forma de sopas ou caldos, diminuindo a biodisponibilidade do mesmo.5,26 É importante citar o papel da vitamina $\mathrm{C}$ na absorção de ferro, podendo a carência dessa vitamina contribuir potencialmente para o surgimento da anemia ferropriva. Costa et al. 27 apontam para uma correlação entre o efeito da suplementação da vitamina $C$ no estado hematológico em seres humanos.

As prevalências de inadequação de ferro foram bastante elevadas em todas as áreas geográficas. Existe uma tendência à diminuição da prevalência de inadequação à medida que aumenta a idade da criança, a qual corrobora com a prevalência de anemia encontrada no Estado, que inversamente diminui com o aumento da idade. ${ }^{9}$ A anemia é considerada atualmente um sério problema de saúde pública e está relacionada com altos riscos de morbidade e mortalidade em crianças. 9

$\mathrm{O}$ zinco possibilita várias funções bioquímicas, uma vez que é componente de inúmeras enzimas, incluindo as do sistema nervoso central. Esse mineral atua na divisão celular, expressão genética e processos fisiológicos de crescimento e desenvolvimento. A suplementação do zinco tem impacto positivo na prevenção da morbidade e mortalidade em crianças. ${ }^{28}$ Apesar de sua importância, os resultados deste estudo também apresentaram elevadas prevalências de inadequação desse nutriente em todas as faixas etárias e áreas geográficas. Alguns fatores poderiam explicar esses resultados: para as crianças menores, considerando que ao nascimento não há reserva de zinco suficiente para prover suas necessidades, o nutriente tem de ser suprido pela alimentação, existindo um potencial para a ocorrência de deficiência desse mineral no início da infância. ${ }^{29}$ Nas faixas de idade mais elevadas, onde se verificam as maiores inadequações, o consumo de leite, carne vermelha, fígado e ovos, consideradas as melhores fontes de zinco, é diminuído. 5

O fato de o zinco estar presente na dieta também não garante a sua utilização pelo organismo, pois a ocorrência da deficiência desse mineral está associada à presença de outros constituintes dietéticos que inibem (fitatos) ou facilitam (cisteína e histidina) sua absorção. 29

Apesar de ter sido demonstrado que o leite, reconhecidamente a maior fonte de cálcio, constitui o alimento mais consumido pelas crianças no Estado de Pernambuco, 5 o percentual de crianças que estavam abaixo da ingestão adequada foi bastante elevado, variando de $23,4 \%$ a $81,9 \%$ entre as faixas etárias, com valores bem mais elevados no Interior Rural (Resultados não apresentados em tabelas). Castro et al., 30 estudando a dieta dos pré-escolares de Viçosa, Minas Gerais, encontraram baixa mediana de ingestão de cálcio contrastando com a ingestão freqüente de leite e derivados, atribuindo o fato ao pequeno porcionamento desses alimentos oferecidos às crianças.

Os resultados mostraram que à medida que aumentava a idade das crianças, diminuía a mediana de ingestão do cálcio, o que tem relação com a mudança no hábito alimentar, uma vez que o consumo de leite diminui gradativamente quando outros alimentos são incorporados à alimentação da criança; entretanto esses alimentos não representam boas fontes de cálcio.5,7 Tais resultados são preocupantes, pois as crianças estão em fase de crescimento rápido e retenção de cálcio para a formação óssea. O risco de desenvolver osteoporose na vida adulta depende da massa óssea alcançada na idade jovem, e as doenças do adulto podem e devem ser prevenidas desde a infância. ${ }^{23}$

\section{Considerações finais}

Os resultados deste trabalho mostram que o consumo alimentar das crianças do Estado de Pernambuco apresenta déficit de energia, de macro e micronutrientes, sobretudo no Interior Rural, onde as prevalências de inadequação foram mais elevadas. Houve importantes riscos de inadequação de ferro, zinco e vitamina A, que são essenciais para o crescimento e desenvolvimento saudáveis das crianças. O consumo 
mediano de proteína estabeleceu-se acima dos valores de referência, apesar de também haver inadequação desse nutriente. As maiores inadequações do consumo alimentar se encontram nas crianças acima de um ano, fase em que deveriam estar recebendo os mesmos alimentos da família, em maior quantidade e variedade.

Ressalta-se a importância da realização de novos inquéritos de consumo alimentar, de base populacional, que impulsionem o monitoramento nutricional das crianças. Com as novas referências, Dietary Reference Intakes, é necessário que a metodologia utilizada nos inquéritos de consumo

\section{Referências}

1. Giugliani ERJ, Victora CG. Alimentação complementar. J Pediatr. (Rio J) 2000; 76 Supl 3: 253-62.

2. Evangelista J. Alimentos: um estudo abrangente. São Paulo: Atheneu; 2000.

3. Batista Filho M. Alimentação, saúde e nutrição. In: Rouquayrol MZ, Almeida Filho N. Epidemiologia \& saúde. 5.ed. Rio de Janeiro: MEDSI; 1999. p. 353-74.

4. Monteiro CA, Benício MHA, Iunees R, Gouveia, NC, Cardoso MAA. Evolução da desnutrição infantil. In: Monteiro CA. Velhos e novos males da saúde no Brasil: a evolução do país e de suas doenças. São Paulo: HUCITEC; 1995. p. 93-144

5. Farias Júnior G, Osório MM. Padrão alimentar de crianças menores de 5 anos no Estado de Pernambuco. Rev Nutr. 2005; 18: 793-802.

6. Assis AMO, Barreto ML. Condições de vida, saúde e nutrição na infância em Salvador. Brasília, DF: INAN; 2000

7. Oliveira MA, Osório MM. Consumo de leite de vaca e anemia ferropriva na infância. J Pediatr. (Rio J) 2005; 81: 361-7.

8. Batista Filho M, Romani SAM. Alimentação, nutrição e saúde no estado de Pernambuco: espacialização e fatores socioeconômicos. Recife: Instituto Materno Infantil de Pernambuco, IMIP; 2002.

9. Osório MM, Lira PI, Ashworth A. Factors associated with $\mathrm{Hb}$ concentration in children aged 6-59 months in the State of Pernambuco, Brazil. Br J Nutr. 2004; 91: 307-15.

10. Cavalcante AAM, Priore SE, Franceschini SCC. Estudos de consumo alimentar: aspectos metodológicos gerais e o seu emprego na avaliação de crianças e adolescentes. Rev Bras Saúde Matern Infant. 2004; 4: 229-40.

11. Pernambuco. Governo. Crianças e adolescentes em Pernambuco: saúde, educação e trabalho. Recife: UNICEF; 1992.

12. BEMFAM (Sociedade Civil Bem-Estar Familiar). Pesquisa nacional sobre demografia e saúde: 1996. Rio de Janeiro; 1996 alimentar seja ajustada a esse modelo de interpretação. Também se faz necessário a utilização de tabelas de composição centesimal de nutrientes condizentes com os alimentos regionais e para esse fim, atualmente, o Ministério da Saúde recomenda a Tabela Brasileira de Composição de Alimentos. 31

Espera-se que esses resultados possam servir de subsídios para implementação de políticas de alimentação e nutrição no Estado, atendendo às reais necessidades da população de menores de 5 anos, como forma de prevenir os principais distúrbios nutricionais.
13. Philippi ST, Szarfarc SC, Latterza AR. Virtual Nutri [programa de computador]. Versão 1.0 for Windows. São Paulo: Departamento de Nutrição da Faculdade de Saúde Pública, USP; 1996.

14. FIBGE (Fundação Instituto Brasileiro de Geografia e Estatística). Tabelas de composição de alimentos. Rio de Janeiro; 1977.

15. Ministério da Saúde. Secretaria de Políticas de Saúde. Coordenação Geral da Política de Alimentação e Nutrição. Alimentos regionais brasileiros. Brasília, DF; 2002. (Série F: Comunicação e Educação em Saúde, n. 21.)

16. Institute of Medicine. National Research Council. Dietary reference intakes for energy, carbohydrate, fiber, fat, fatty acids, cholesterol, protein, and amino acids (Macronutrients). Washington, DC: National Academy Press; 2002.

17. Institute of Medicine. National Research Council. Dietary reference intakes for vitamin A, vitamin $\mathrm{K}$, arsenic, boron, chromium, copper, iodine, iron, manganese, molybdenum, nickel, silicon, vanadium, and zinc. Washington, DC: National Academy Press; 2002.

18. Institute of Medicine. National Research Council. Dietary reference intakes for vitamin $\mathrm{C}$, vitamin $\mathrm{E}$, selenium, and carotenoids. Washington, DC: National Academy Press; 2002.

19. Institute of Medicine. National Research Council. Dietary reference intakes for calcium, phosphorus, magnesium, vitamin D, and fluoride. Washington, DC: National Academy Press; 1999.

20. Marchioni DML, Slater B, Fisberg RM. Aplicação das Dietary reference intakes na avaliação da ingestão de nutrientes para indivíduos. Rev Nutr PUCCAMP. 2004; 17: 207 16.

21. Ferro-Luzzi A. Individual food intake survey methods. International Scientific Symposium on Measurement and Assessment of Food and Deprivation and Undernutrition. Rome; 2002.

22. FIBGE (Fundação Instituto Brasileiro de Geografia e Estatística). Estudo Nacional das Despesas Familiares. Consumo alimentar e antropometria. Rio de Janeiro; 1977. 
23. Williams SR. Fundamentos de nutrição e dietoterapia. 6.ed. Porto Alegre: Artes Médicas, 1997.

24. Diniz AS, Santos LMP. Hipovitaminose A e xeroftalmia. J Pediatr (Rio J) 2000: 76 (Supl 3): 311-22.

25. Semba RD, Bloem MW. The anemia of vitamin A deficiency: epidemiology and pathogenesis. Eur J Clin Nutr. 2002; 56: 271-81.

26. Cruz GF, Santos RS, Carvalho CRG, Moita GC. Avaliação dietética em creches municipais de Teresina, Piauí, Brasil. Rev Nutr. 2001; 14: 21-32.

27. Costa MJC, Terto ALQ, Santos LMP, Rivera MAA, Moura LSA. Efeito da suplementação com acerola nos níveis sangüíneos de vitamina $\mathrm{C}$ e de hemoglobina em crianças pré-escolares. Rev Nutr. 2001; 14: 13-20.
28. Mafra D, Cozzolino SMF. Importância do zinco na nutrição humana. Rev Nutr. 2004; 17: 79-87.

29. Lönnerdal B. Dietary factors influencing zinc absorption. J Nutr. 2000; 130: 1378-85.

30. Castro TG, Novaes JF, Silva MR, Costa NMB, Francheschini SCC, Tinôco ALA, Leal PFG. Caracterização do consumo alimentar, ambiente socioeconômico e estado nutricional de pré-escolares de creches municipais. Rev Nutr. 2005; 18: 321-30.

31. Universidade de Campinas. Tabela Brasileira de Composição de Alimentos. Versão II. 2. ed. Campinas, SP; 2006.

Recebido em 21 de junho de 2006

Versão final apresentada em 19 de novembro de 2006

Aprovado em 20 de dezembro de 2006. 